\title{
Waveform-based gradient method for estimating hypocenter mechanism before observing aftershocks
}

\author{
Louise CORDRIE ${ }^{1,2}$ and Hitoshi MIKADA ${ }^{1}$ \\ ${ }^{1}$ Dept. of Civil and Earth Res. Eng., Kyoto University \\ ${ }^{2}$ On leave from EOST, School and Observatory of Earth Science, Strasbourg University
}

\begin{abstract}
The location of the hypocenter of an earthquake as well as the determination of the source parameters have shown some limitations in the past. The actual studies often neglect the tailing of the waveform to concentrate on the first waves arrivals and the inversion methods often need a precise distribution of aftershocks to locate the fault plane. Using former models of fault slip propagation, we produce synthetic waveforms related to different fault geometries. The inversion method will be adapted to analyze the total waveform and, using a higher number of seismograms, will give back the source parameters of the studied earthquake. The different geometries of slip models give back precise waveforms and the flexibility of the code should be an advantage to future inversion processes of earthquake location. The improvements of the fault models and the inversion method are discussed through this paper.
\end{abstract}

Key words: fault, double-couple moment tensor, P-wave radiations, S-wave radiations, source mechanism, inversion.

\section{INTRODUCTION}

There are a lot of examples in numerical estimation of physical mechanisms of earthquakes. Due to the limitation in the number of available seismographs in the world in the past, the estimation either using CMT solutions or Monte-Carlo type inversion schemes has been dominant in the literature as in the two major papers of Tarantola (1984) $^{4)}$ and Kanamori (1986) ${ }^{3)}$. The former assumes a hypocenter of an earthquake to be a point source and estimates the mechanism of the earthquakes (strike-slip, normal or reverse), although the fault plain of the earthquake should have some finite physical dimensions. The latter assumes the hypocenter as a group of fault planes to cover the finite-scale earthquake fault and gives the idea which part of the fault plane has worked as asperities. The methods have been used to quickly estimate what kind of earthquake has taken place in a short period after the occurrence of each earthquake but they have limitations. Indeed, the former models only use the initial quarter to half the wavelength and ignores any tailing waveforms. The latter assumes the location of a fault plane that caused the earthquake using the distribution of aftershocks. Those have been remaining shortcomings, since we need to watch the distribution of aftershocks carefully to identify the fault location.

Thanks to the increasing number of seismometers we are convinced that we may be able to improve the situation using some leading-edge technologies such as wave theories. After the development of a 2D slip fault model, we try to estimate the location of hypocenter based on earthquake waveforms observed by an increased number of seismometers.

This paper tackles the first part of the research concerning the modelling of the 2D slip fault. This paper begins with a presentation of the fault models based on Seismic source theory chapter by Madagaria (2009) followed by the results of waveforms induced by a moment tensor source. Then after discussing of future model improvements we finally introduce the inversion method, presented in the Slip inversion chapter by S. Ide (2009) and developed in Tarantola (1984) ${ }^{4}$ and Kanamori $(1986)^{3)}$ papers, which will enable the determination of source parameters.

\section{METHOD}

The construction of a fault model is carried out in several steps. The principles are explained in detail in the Seismic source theory chapter by Madagaria $(2009)^{1)}$. A fault plane slip is equivalent to a group 
of discrete points, in a 2D grid, by which the rupture is propagated and which are defined by a slip amplitude, a rake angle and a time function. Those parameters define the source mechanism, which is, in a second time, determined by the least-square inversion method by comparing the model with real data. Each of these points corresponds to a point moment tensor source, so it generates radiations in the space surrounding it and the superposition of the radiations from the set of point source gives the final waveform observed at any position of the space.

\section{(1) Point source model}

The first step of the model is the understanding of the radiations produced by one point source in a homogeneous medium (figure 1). The equation $\left(\right.$ Madagaria-2009) ${ }^{1)}$ of a moment tensor source is:

$$
u_{k}(x, t)=\sum_{i j} G_{k i, j}\left(x, t \mid x_{0}, t\right) * M_{i j}(t)
$$

Where $G_{i j}\left(x, t \mid x_{0}, t\right)$ is the Green function for a force in the direction $i$, located at a point $x_{0}$ at a time $t$ and $M_{i j}(t)$ is the moment of this point force. Then the $\mathrm{P}$ and $\mathrm{S}$ waves induced by the source are producing at a distance $R$ the following displacements ${ }^{1)}$ :

$$
\begin{aligned}
& u_{i}^{p}(R, t)=\frac{1}{4 \Pi \rho \alpha^{3}} \frac{1}{R} \sum_{j k} R_{i j k}^{p} \dot{M}_{j k}\left(t-\frac{R}{\alpha}\right) \\
& u_{i}^{S}(R, t)=\frac{1}{4 \Pi \rho \beta^{3}} \frac{1}{R} \sum_{j k} R_{i j k}^{S} \dot{M}_{j k}\left(t-\frac{R}{\beta}\right)
\end{aligned}
$$

Where $R_{i j k}^{p}$ and $R_{i j k}^{S}$ are the radiation patterns of the waves and $\dot{M}_{j k}$ is the moment rate of the source.

During a fault slip earthquake, the tensor is generally a double-couple moment tensor with two point forces of direction $i$ and two point forces of direction j.

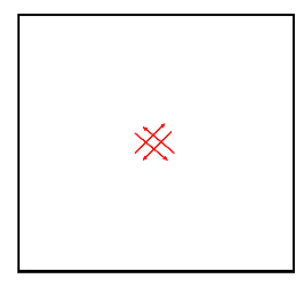

Figure 1: Double couple moment tensor source centered on a 2D fault plane.
The finite source model corresponds to a distribution of double-couple moment tensor source. The total waveform obtained at an observation point $x$, is the result of the superposition of the radiations induced by each of those point sources ${ }^{1)}$ :

$$
\begin{aligned}
u_{i}(x, t)=\int_{0}^{t} \int_{S_{x_{0}}} \mu\left(x_{0}\right) \Delta u_{j}\left(x_{0}, \tau\right) \\
\quad \times G_{i j, k}\left(x, t \mid x_{0}, \tau\right) n_{k}\left(x_{0}\right) d^{2} x_{0} d \tau
\end{aligned}
$$

$\Delta u_{j}\left(x_{0}, \tau\right)$ corresponds to the amplitude of the slip at the $x_{0}$ point source and $\mu\left(x_{0}\right)$ its elastic parameters.

\section{Linear propagation:}

The first and simple model is the Haskell's one ${ }^{1)}$, it considers a linear rupture front propagation with a constant slip amplitude (figure 2). At each time step a line of double-couple moment tensor source points appears at a distance $d=V r * t$ of the initial source and slip with an intensity of D.

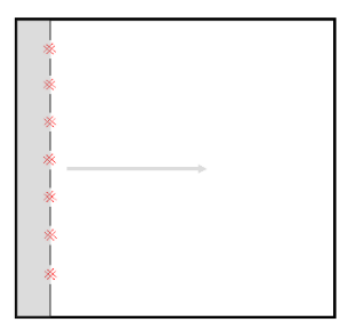

Figure 2: Propagation of the front rupture on a fault plane through a linear distribution of the moment tensor sources.

The linear propagation from Haskell model includes a directivity effect, computed in the source-time function. The angle $\theta$ between the observation point and the propagation axis influences the duration of the pulse $T_{M}$. It is interpreted by the convolution of 2 boxcars where $\tau_{r}$ is the rising time 1):

$$
\Omega(\theta, 0, t)=M_{0} \operatorname{boxcar}\left[t, T_{M}\right] * \operatorname{boxcar}\left[t, \tau_{r}\right]
$$

\section{(2) Finite fault model ${ }^{1)}$}


This way a point localized on the propagation axis will undergo more intense displacements than a point localized at a same distance but in an orthogonal direction.

Circular propagation:

To make a more realistic model, a circular propagation can be used: at each time step, each point with a radius, from an initial central source, $R=$ $V r * t$ constitute a new double-couple moment tensor source (figure 3).

\section{RESULTS}

The previous fault slip geometries can be modeled using a $\mathrm{C}++$ and Matlab codes. The elastic parameters, fault parameters, time functions and green functions are input in the code and it gives back the induced waveform at any observation point as well as a 2D map of the displacement intensity. We first apply the calculations to an infinite homogeneous and elastic medium. The time and space scale are chosen in a way to make realistic model as well as short calculation time.

\section{(1) Point source model}

An earthquake source is defined by a time-function which gives the wavelet shape, by a position and by one or several directions. For a fault slip, a realistic wavelet shape is given by the Gaussian function but the triangle and boxcar function can also be used
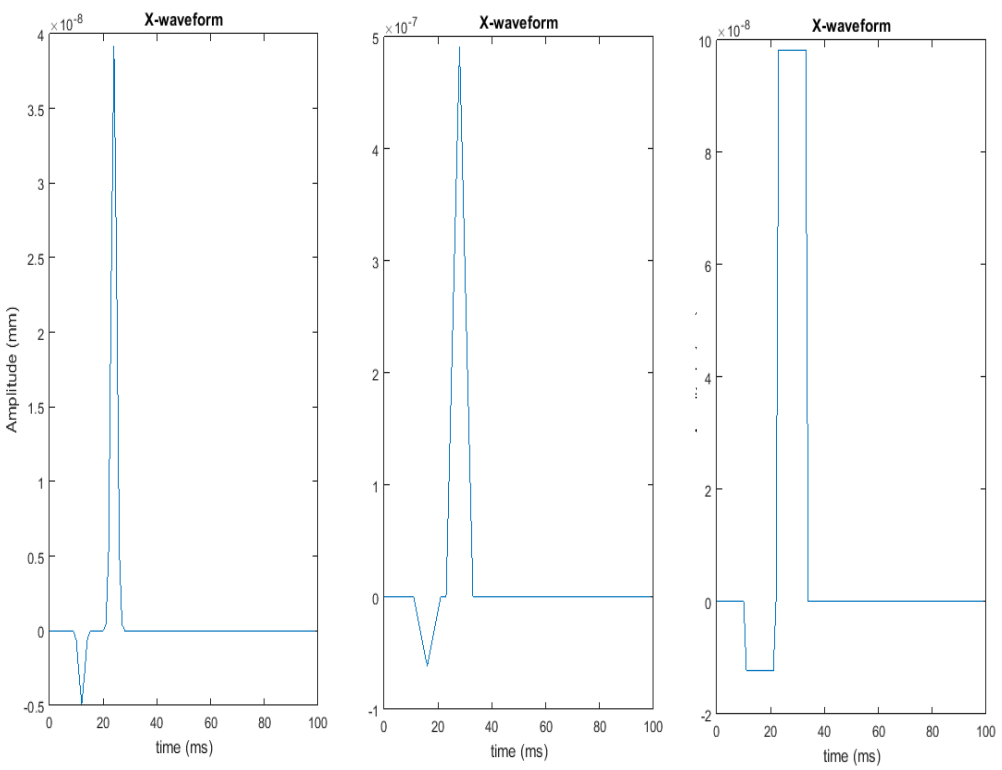

Figure 4: Waveforms induced by a Gaussian (left), Triangle (middle) and Boxcar (right) time-functions.

depending of the model. The point source green function separates the radiations in three well known categories: The $\mathrm{P}$-wave radiations which are appearing on the direction of propagation and the Swave radiations which are appearing on the two other directions since it includes the vertical component SV and the horizontal component $\mathrm{SH}$.

The waveform induced by a double-couple point source model is a combination of the initial wavelet and those radiation directions. The waveform obtained at an arbitrary point of the infinite medium

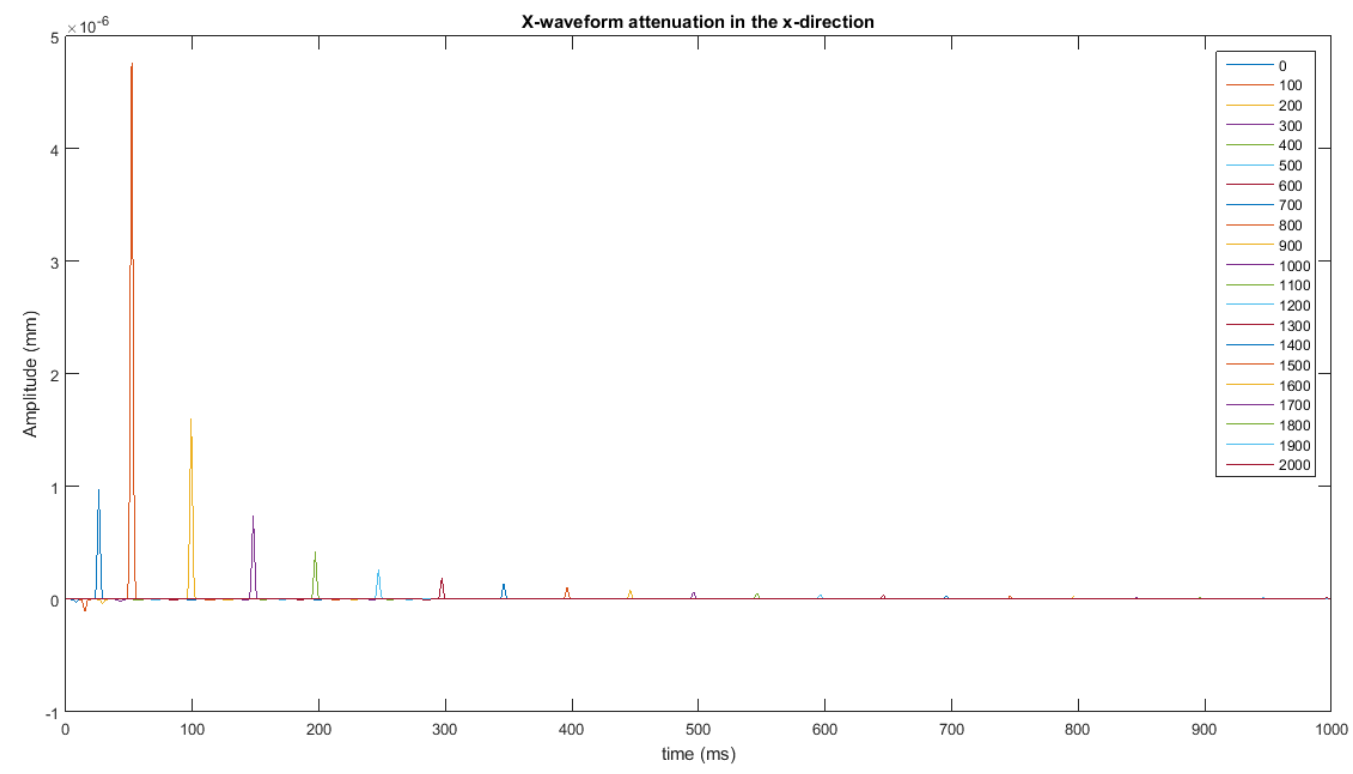

Figure 5: Waveform attenuation along the $X$-axis (the legend corresponds to the distance of the observation point from the source). 
for different source-time functions will have different shapes (figure 4).

The radiations through the medium are ongoing a spherical attenuation due to the geometrical expansion of the wave front with distance. This attenuation is observed on the display of waveforms at increasing distances from the source (figure 5).

\section{(2) Fault model}

A simple representation of a fault is the creation of a 2D-matrix where each point has a precise position in the chosen referential system. The sources created at each propagation step of the rupture front are part of this matrix. The radiations are computed in an independent 3D matrix with a different referential system. The transition from one to the other is made by applying translation and rotation transformations to the value of displacements in the three directions. In this way, the dip angle, the depth and other parameters of the fault can easily be changed without needing any recalculations.
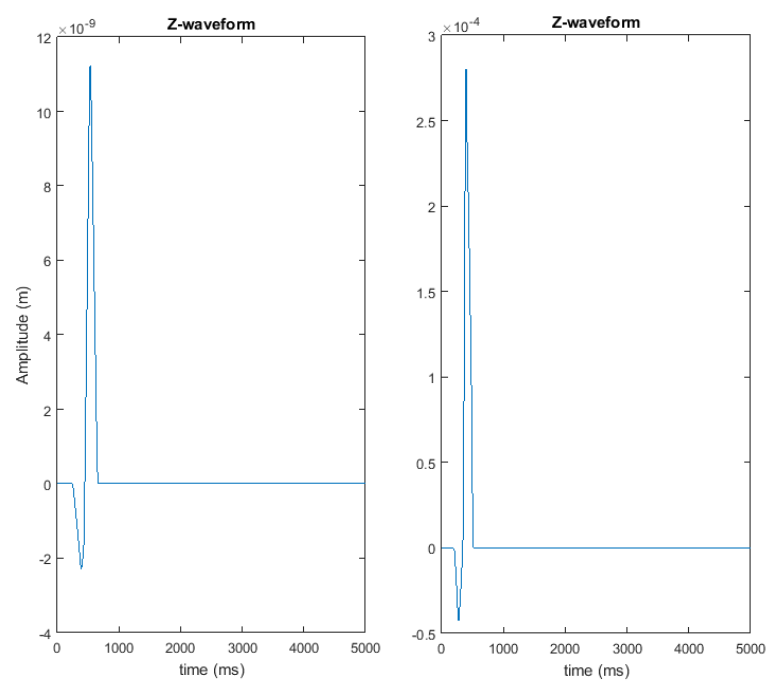

Figure 6: Z-direction waveforms for a linear propagation (left) and circular propagation (right).

The linear and circular propagation models give, at a same arbitrary point for the same fault parameters, different waveforms (figure 6). The two models are based on similar source-time function and parameters which explain the similarity of the waveforms. However, the displacement intensities are different because they are due to the superposition of the sources and their geometry of propagation. The directivity effect of the linear model (figure 7), produces a difference of 2 orders of intensity and very different waveform shapes.

In the study of earthquake's source parameters, the determination of the direction of the fault plane regarding the focal mechanism is a crucial step. Especially the differentiation of two perpendicular strike-slip fault planes based on the waveforms. A waveform comparison was applied for different fault angles at a same observatory point (figure 8). The subtraction of two waveforms gives another timefunction. The sum of the absolute values of each time-function can be called a subtraction residual. The closest angle from the computed point is the one presenting the smallest residual, the written code gives at the end an order of the angles reproducing the best the initial waveform (figure 9). This shows the influence of the rake angle. A similar process can be lead for the dip angle and the other source parameters.
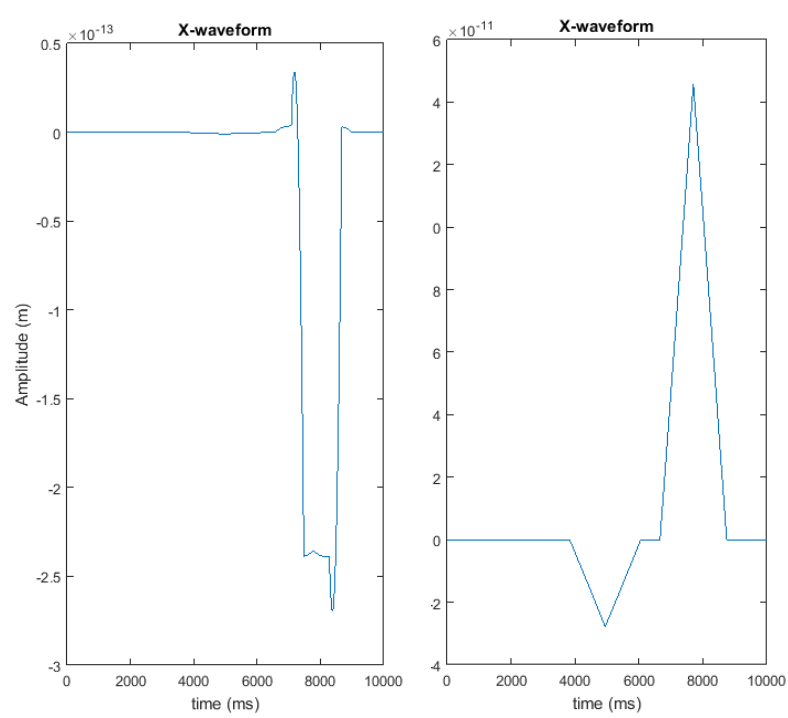

Figure 7: Directivity effect on two waveforms at points localized $25000 \mathrm{~m}$ away from the fault along the perpendicular axis (left) and the rupture axis (right).

\section{DISCUSSION}

The models discussed previously are far from real earthquake's fault geometry. Inhomogeneities should be added to the model, which will enable more complex slip propagation. An efficient method to increase the accuracy of the model is the use of the multi-time window method presented in the Slip inversion chapter by S.Ise $(2009)^{2)}$, where the fault 2D matrix is not subdivided in points but in subfaults. The sub-faults are made of a group of points which have their own time-function and source parameters.

Once the model can be compared to the real data, the direct problem is completed and the indirect problem 
begins. The waveform comparisons realized previously are just simplified trials of the problem of inversion. The code will be developed to make a more precise localization of the source through the least-square method. But also by searching for the
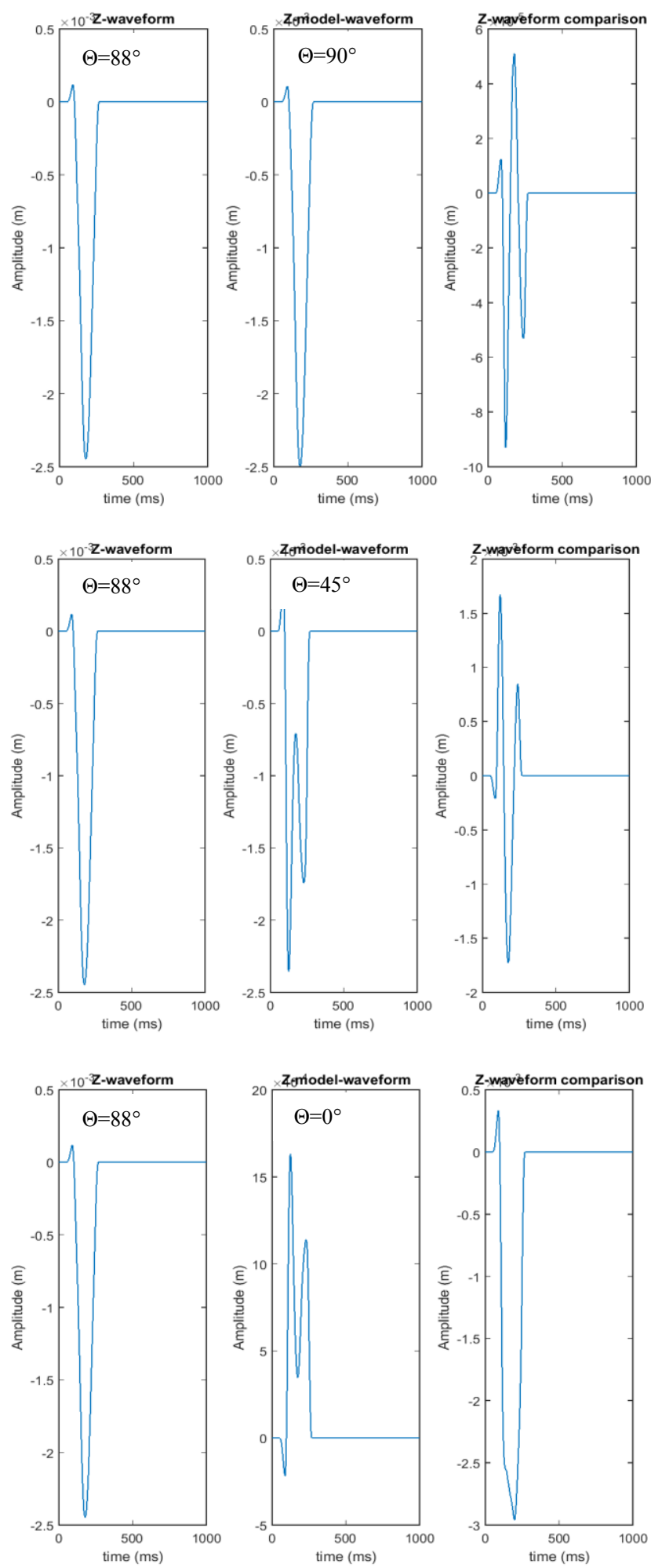

Figure 8: $88^{\circ}$ fault angle waveform compared to $90^{\circ}, 45^{\circ}$ and $0^{\circ}$ fault angle waveforms.

source parameters which minimize the residual. The model will get closer to the real geometry and should enable a precise determination of the source mechanism.

As discussed by Tarantola (1984), the residual waveform in the modeled compared to the observed could be propagated back to the locations in the model space as the gradient field to update parameters for the next iteration in the inverse procedure. This iterative inverse procedure is called full waveform inversion (FWI). Since the present results clearly demonstrated that the waveforms generated by the two faults plane intersecting with each other with the conjugate angle are different from each other, an approach similar to FWI could be applied to estimate fault rupture parameters such as the location of the fault plane, distribution of dislocations and the distributed source time functions over the fault plane.

\section{CONCLUSIONS}

The models of fault slip propagation must be chosen in function of the inversion process which will be applied to them. Since the geometry of an event can achieve so different shape, the first rupture model should be simple. Moreover, to work on an inversion based on a high number of input waveforms, this model should also be flexible enough. The models developed now must be improved following the directions suggested in the discussion part before reaching the process of inversion. 


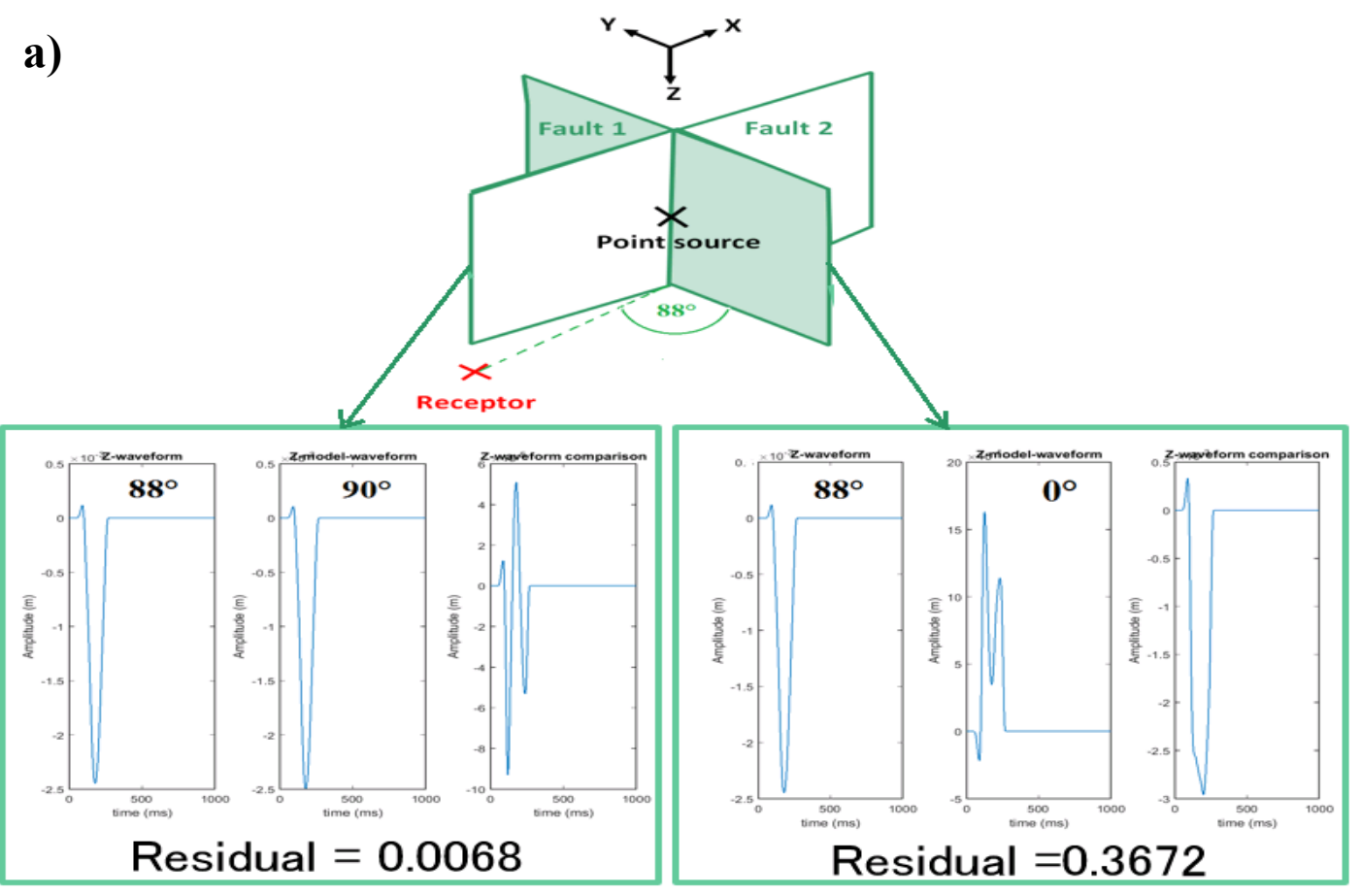

b)

\begin{tabular}{|l|l|l|l|}
\hline Angle & X-direction & Y-direction & Z-direction \\
\hline $90^{\circ}$ & 0.007706 & 0.003344 & 0.006808 \\
\hline $70^{\circ}$ & 0.029313 & 0.085277 & 0.086441 \\
\hline $45^{\circ}$ & 0.163924 & 0.291746 & 0.154298 \\
\hline $20^{\circ}$ & 0.309434 & 0.222399 & 0.186272 \\
\hline $0^{\circ}$ & 0.350777 & 0.006165 & 0.367158 \\
\hline
\end{tabular}

Figure 9: a) Waveforms and residual for the $0^{\circ}$ and $90^{\circ}$ angles. b) Waveform subtraction's residual for an initial $88^{\circ}$ fault angle waveform (The $0^{\circ}$ angle corresponds to the $X$-axis and the $90^{\circ}$ angle corresponds to the Y-axis).

\section{REFERENCES}

1) Madagaria R., Schubert G. and Kanamori H., 2009, Seismic Source Theory, Treatise on Geophysics, Vol.4: 59-82.

2) Ide S., Schubert G. and Kanamori H., 2009, Slip Inversion, Treatise on Geophysics, Vol.4: 193-223.

3) Kikuchi M., Kanamori H., 1986. Inversion of complex body waves-II. Phys. Earth Planet. Inter., 43: 205-222.

4) Tarantola A., 1984. Inversion of seismic reflection data in the acoustic approximation. Geophysics, Vol. 49, No. 8: 1259-1266. 